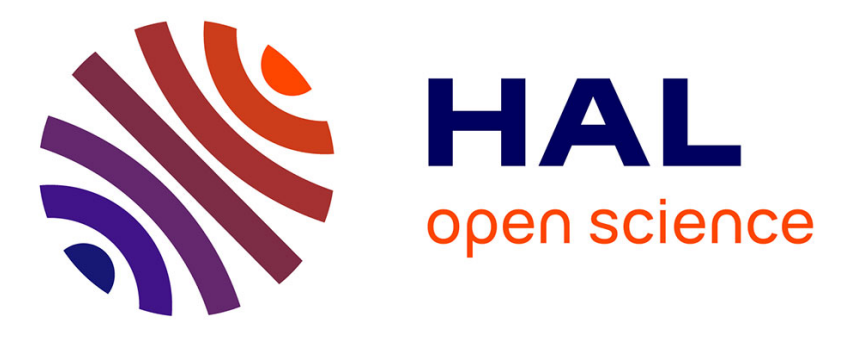

\title{
Inter-Hemispheric Remapping between Arm Proprioception and Vision of the Hand is Disrupted by Single Pulse TMS on the Left Parietal Cortex Lilian Fautrelle, Mathieu Gueugnon, Guillaume Barbieri, François Bonnetblanc
}

\section{To cite this version:}

Lilian Fautrelle, Mathieu Gueugnon, Guillaume Barbieri, François Bonnetblanc. Inter-Hemispheric Remapping between Arm Proprioception and Vision of the Hand is Disrupted by Single Pulse TMS on the Left Parietal Cortex. Brain and Cognition, 2013, 82 (2), pp.146-151. 10.1016/j.bandc.2013.03.002 . lirmm-00805192

\section{HAL Id: lirmm-00805192 \\ https://hal-lirmm.ccsd.cnrs.fr/lirmm-00805192}

Submitted on 27 Mar 2013

HAL is a multi-disciplinary open access archive for the deposit and dissemination of scientific research documents, whether they are published or not. The documents may come from teaching and research institutions in France or abroad, or from public or private research centers.
L'archive ouverte pluridisciplinaire HAL, est destinée au dépôt et à la diffusion de documents scientifiques de niveau recherche, publiés ou non, émanant des établissements d'enseignement et de recherche français ou étrangers, des laboratoires publics ou privés. 
Inter-hemispheric remapping between arm proprioception and vision of the hand is disrupted by single pulse TMS on the left parietal cortex.

Lilian Fautrelle ${ }^{1,2}$, Mathieu Gueugnon ${ }^{1,2}$, Guillaume Barbieri ${ }^{1,2}$, François Bonnetblanc $^{1,2,3^{*}}$

${ }^{1}$ Université de Bourgogne, Dijon, Campus Universitaire, Unité de Formation et de Recherche en Sciences et Techniques des Activités Physiques et Sportives.

${ }^{2}$ Institut National de la Santé et de la Recherche Médicale, Unité 1093, Cognition, Action et Plasticité Sensori-Motrice, F-21078 Dijon, France.

${ }^{3}$ Université Montpellier 2 - LIRMM - DEMAR Team, 161 Rue Ada, F-34095 Montpellier Cedex 5

*Corresponding author: francois.bonnetblanc@u-bourgogne.fr 
Abstract

Parietal cortical areas are involved in sensori-motor transformations for their respective contralateral hemifield/body. When arms of the subjects are crossed while their gaze is fixed straight ahead, vision of the hand is processed by the hemisphere ipsilateral to the arm position and proprioception of the arm by the contralateral hemisphere. It induces interhemispheric transfer and remapping. Our objective was to investigate whether a single pulse TMS applied to the left parietal cortical area would disturb interhemispheric remapping in a similar case, and would increase a simple reaction time (RT) with respect to a control single pulse TMS applied to the frontal cortical area. Two LED were superimposed and located in front of the subjects on the saggital axis. Subjects were asked to carefully fixate on these LED during each trial. The lighting of the red LED was used as a warning signal. Following the green one was illuminated after a variable delay and served as a go-signal. The hand for the response was determined before the start of each trial. TMS was applied to the left parietal, the left frontal cortical areas, or not applied to the subject. Results revealed that: (1) Irrespective of its location, single pulse TMS induced a non-specific effect similar to a startle reflex and reduced RT substantially (15 ms on average) with respect to a control condition without TMS (mean value $=153 \mathrm{~ms}$ ). (2) Irrespective of TMS, RT were shorter when the right or the left hand was positioned in the right visual hemi-field (i.e. normal and crossed positions respectively). (3) Finally, RT increased when single pulse TMS was applied to the left parietal area and when hands were crossed irrespective of which hand was used. We concluded that interhemispheric sensori-motor remapping was disrupted by a single pulse TMS that was applied to the left parietal cortex. This effect was also combined some visual attention directed towards the hand located on the right visual hemi-field.

Keywords: Interhemispheric transfer; remapping; transcranial magnetic stimulation (TMS); left parietal area; simple reaction time (RT); startle reflex. 


\section{Introduction}

Various sensory modalities converge and integrate to the CNS across the parietal cortex (Head and Holmes 1911). In this associative part of the brain, integrative processes unify percepts of the body and/or environment in a common reference frame in order to act (Andersen 1995). More specifically, the parietal cortex is involved in many sensori-motor transformations especially, in sensory transformations from proprioceptive and visual to motor coordinates (Duhamel et al. 1992, 1997, Cohen and Andersen 2002, Buneo 2002). During hand actions, it has also been shown that the posterior parietal cortex updates the continuous postural changes, through proprioception, in order to allow a spatial remapping of the representation of the body (Lloyd et al. 2003, Bolognini and Maravita 2007).

As each hemisphere deals with the opposite hemi-field and hemi-body, this continuous update can become more complex when one sensory modality is displayed in one hemi-field or hemibody and another in the opposite (Azanon et al. 2010, Heed and Roder 2010, Overvliet et al. 2011, Buchholz et al. 2012). This particular situation occurs for instance when we are looking straight ahead and respond to a visual stimulus located in the left hemifield with the right arm. In this case, the visual stimulus is processed in the right hemisphere while proprioceptive inputs are processed in the left hemisphere (Marzi et al. 1991, 1998). These situations require some level of inter-hemispheric transfer for both kind of information and the representation of the visual stimulus processed by the right hemisphere has to be remapped into the proprioceptive system in the left hemisphere. In a more subtle case, when arms are just crossed, and the gaze is fixed straight ahead, visual information about the hand position and proprioception of the arms are processed by different hemispheres. During hand goal directed movements, it has been demonstrated that the vision of the hand prior to the movement was processed to improve the rate of terminal error (Rossetti et al. 1995). By contrast, when no hand displacement is required like for instance in a simple visuo-manual reaction, it is 
unknown whether the vision of the hand in the opposite hemifield is still processed. The brain may deal with a single sensory modality and no inter-hemispheric remapping would be required when arms are crossed in this simple task. Consequently inter-hemispheric transfer is believed to increase RT at least due to transmission delays along the corpus callosum (Overvliet et al. 2011). In principle accordance, we investigated whether a single pulse TMS applied to the left parietal cortex would increase simple visuo-manual RT when arms are crossed. Indeed, cortically mediated functions can be inhibited with single pulse TMS (see Wassermann et al. 2002 for a review). To alleviate attentional effects as well as to demonstrate that disruption of inter-hemispheric remapping was specific to the parietal cortex, we located the visual go-signal in the saggital axis. In consequence, the visual spatial attention was focused on the central stimulus and also balanced between the right and left hemifields independently of the position of the arm. Consequently, an increase of RT with a single pulse TMS applied over the parietal cortex in the arms crossed condition would indicate impairments in the inter-hemispheric remapping rather than impairments in attention processes with respect to a normal uncrossed condition. In addition, we applied a single pulse TMS to the frontal cortex as a control condition. The frontal cortex is considered to be involved in movement selection (Petrides and Milner 1982, Goldman-Rakic 1987) but its implication in attention processes and its connectivity with the parietal cortex have also been demonstrated and recently confirmed (Thiebaut de Schotten et al. 2005). Concerning this frontal control TMS condition, the left Dorsolateral Prefrontal Cortex (DLPFC) is also known to be an important region for the performance in Go/No-Go tasks involving executive functions (e.g. van den Heuvel et al., 2013, Bermpohl et al., 2005). Consequently, a simple reaction time paradigm with only go-signals (and without no-go signal), for which the motor responses was selected before each trial, allowed us to limit as much as possible the 
participation of executive functions and the involvement of DLPFC. Therefore, TMS applied other this area should not perturb information processing and induce increased reaction times. To investigate our hypothesis, we used a simple RT paradigm and designed a task in which subjects had to respond to a single central visual stimulus as fast as they could with one hand. The position of both hands were also varied in the visual field by crossing them. Importantly, the task was also performed without any cueing or manipulation of the stimulus-response compatibility. Consequently, no visual attention or movement selection constraint was introduced in the task and we sought to limit as much as possible the processes superimposed or mixed with sensori-motor transformations. More specifically, we hypothesized that if the hands position in the opposite visual field induces some level of inter-hemispheric remapping between information relative to the vision of the hand and proprioception of the arms, we should observe an increase of the RT for both hands when a single pulse TMS is applied to the left parietal area. 


\section{Methods}

\subsection{Participants}

Nine healthy volunteers (all right handed as assessed by the Edinburgh Handedness Inventory, Olfield, 1971) participated to the experiment. They were all males, $27 \pm 2$ years old (range $=[24 ; 35]$ ) and had no history of previous neurological diseases. The study conformed to the Code of Ethics of the World Medical Association (Declaration of Helsinki, 18 July, 1964) and the general procedure was approved by the local ethics committees.

\subsection{Task and procedures}

Participants sat comfortably in front of two superimposed LED of different colors (red and green) fixed on a table at a distance of $60 \mathrm{~cm}$ from the body in the sagittal axis and $45 \mathrm{~cm}$ below the eyes. The participants were instructed to keep their eyes on these LED from the lighting of the red LED to the end of the recording. Two switches were positioned on each side of the participant (distant $=40 \mathrm{~cm}$ from each other) and located at $45 \mathrm{~cm}$ from the body (Figure 1). Motor responses consisted in releasing one switch by raising their index finger. The red LED served as the warning signal. The green LED represented the "go-signal" prompting the participant to raise one index finger(s) as fast as possible. The time elapsed between the warning signal and the "go-signal", i.e. the stimulus onset asynchrony (SOA), was randomly varied between each trial $(\mathrm{SOA}=2,2.5,3,3.5,4$ and 4.5 seconds). The duration between each trial was comprised between 5 and 10 seconds.

Please insert figure 1 here

Each participant performed the simple reaction time paradigm with their right or left hand (HAND), in two conditions of hand position in the visual field (POSITION), normal or 
crossed together with respect to the saggital axis, for two loci of TMS (TMS) plus a sham condition. In this latter case, TMS was applied to the left parietal, the left frontal areas, or not applied to the subject (sham control condition). In the hands crossed condition, for half trials the right arm was crossed above the left arm and for another half it was the opposite. Before the experiment, for all the subjects, the left parietal and frontal targets for TMS were determined according to the 10-20 system of EEG-electrodes placement and confirmed with an EEG recording cap (64 channels). The 10-20 system is an international and standardized system based on a relationship between the location of a locus on the skull and the underlying brain areas. It is the most widely used method in several disciplines including medicine and neuroscience research to localize the placement of EEG electrodes along the head. This method accounts for some of the variability in subject's skull size by using standardized percentages of the circumference and distances between basic anatomical landmarks (Herwig et al. 2003). Moreover, similar methods have already been used in TMS studies in order to localize the stimulation loci (Herwig et al. 2001, Schutter et al. 2006, Griskova et al. 2007, Desmurget et al. 1999). In our study, to further strengthen our control over the location of the stimulation loci and validate our ability to use the $10-20$ procedures, a neuroradiologist check with MRI the relation between our obtained loci and the cortical areas of our first 4 subjects. For these four participants we verified by MRI, that the localization of the targets marked on the skull of the subjects matched with the parietal and frontal brain areas. Images of brain anatomy were determined for these subjects with a high-resolution MRI scanner. Scans were acquired on a 3 Tesla Magnetom Trio system (Siemens AG, Munich, Germany). We acquired a $\mathrm{T}-1$ weighted high resolution three-dimensional volume (repetition time $=1700 \mathrm{~ms}$, echo time $=2.93 \mathrm{~ms}$, flip angle $=90^{\circ} ; 144$ adjacent axial slices, $1.09 \mathrm{~mm}$ thickness; in-plane voxel size $\left.=1 \times 1 \times 1 \mathrm{~mm}^{3}\right)$. TMS targets were identified by marking the skull of the subjects with a small capsule that produced high-intensity signals on the MRI images. A visual inspection 
was made by a neuroradiologist in order to confirm the correctness of the location. Importantly, we chose this procedure to approximate the parietal and frontal TMS sites because classical procedures involving neuro-navigation devices are ambiguous to determine the TMS sites. Indeed, several approximations linked to neuro-navigation methods are substantial. Inter-individual variability of brain anatomy and precise localizations often require a great expertise in brain anatomy which is often minored, especially when performed on MRI scans (personal experience in dissection of humain brains of the senior author FB supervised by Igor L. Maldonado). For instance, if a template based on an averaged brain anatomy is used to localize the TMS site, it may fall out of the real anatomical landmark. In addition, the expertise required to precisely localize most anatomical landmarks needs a lot of experience in brain anatomy that is not really reached by most experimenters. As such we assume our likely minor lack of accuracy. Finally, despite a minor lack of precision in our method for localization, we found that TMS disrupt interhemispheric transfer for 7/9 subjects. A single-pulse TMS was delivered using a 70-mm eight-shaped coil connected to a Magstim 200 stimulator (Magstim, Whitland, UK). The eight-shaped coil was positioned over the loci of stimulation (P3 and F3) directly marked on the skin of the skull during the 10-20 procedures. The coil was then orientated to deliver antero-posterior directed current to the brain and secured in place throughout the experimental block with a 3 dimensional articulated arm and associated with a custom-made collar that ensured stable positioning of the coil and stable positioning of the subjects head. Moreover, an experimenter visually controlled before and after each trial that the coil was still well above the loci of stimulation and in the same orientation by controlling also the configuration of the $3 \mathrm{D}$ articulated arm for each site.

For each subject we estimated the resting motor threshold. In order to determine the resting motor threshold, we first localize the hand motor areas of the right hand (i.e. in the left hemisphere of the brain). In this way and according to the chosen 10-20 procedures, we 
measured the distance between the nasion and the inion and marked the midpoint between these two points as the vertex position (corresponding to the $\mathrm{Cz}$ position). We then measured $3 \mathrm{~cm}$ laterally from the $\mathrm{Cz}$ point toward the left ear and initially positioned the coil in this position. We moved the coil in $0.5 \mathrm{~cm}$ steps around this area in order to find the 'hot spot', which elicited the largest MEP responses in the right hand when stimulated, and we marked this point as the hand area of the left motor cortex (Boorman et al. 2007).

In a second step, we determined the resting motor threshold by delivering single TMS pulses on the hot spot localized in the left hemisphere of the brain and check MEP in the relaxed contra-lateral hand. Pulses were initially delivered with a very low TMS intensity $(30 \%$ of the stimulator output) and this intensity was gradually increased with steps of $5 \%$ until the occurrence of a muscle twitch in the right hand. Finally, the resting motor threshold was defined as the intensity at which $3 / 6$ MEP were detected. TMS intensity was then set at $120 \%$ of the resting motor threshold for the whole experimental session. Sixteen trials were performed for each 2 HAND x 3 TMS x 2 POSITION conditions with a total of 192 trials per subject. Each TMS x POSITION conditions were performed in two separated blocks of 16 trials that were randomized between subjects. Within each of these TMS x POSITION blocks (performed twice), the eights firsts trials were blocked together for one hand and the eight lasts for the other one. These HAND 8 trials blocks were counterbalanced between each TMS $\mathrm{x}$ POSITION blocks. Positions of the switches were also counterbalanced between the two TMS x POSITIONS blocks of 16 trials (8 for each HAND), in order to measure RT for the same hand with the two different switches and to cancel possible differences in electromechanical delays between them. RT, warning and go signals were synchronized together, recorded at a sampling frequency of $5 \mathrm{kHz}$ and processed with a multichannel analog-todigital converter (Biopac Systems, Inc., Goleta, California). RT values that exceeded twice the standard deviation from the RT mean were excluded from the analysis ( $2 \%$ of all trials). 


\subsection{Data analysis}

Simple RT were submitted to a 2 HAND x 3 TMS x 2 POSITION ANOVA with repeated measures on the 3 factors. Post-hoc analyses were conducted when necessary. All statistical analyses were carried out using an alpha level of 0.05 . Post-hoc analyses were conduced with Fisher's LSD when necessary. 


\section{Results}

For reaction times, results of the ANOVA showed no significant main effect of HAND $(\mathrm{F}(1,8)=0.3, \mathrm{P}=0.6)$, a significant main effect of TMS $(\mathrm{F}(2,16)=51, \mathrm{P}<<0.001)$, no significant main effect of POSITION $(\mathrm{F}(1,8)<<0.001, \mathrm{P}=0.99)$, no significant HAND $\mathrm{x}$ TMS interaction $(\mathrm{F}(2,16)=0.56, \mathrm{P}=0.58)$, a significant HAND x POSITION interaction $(\mathrm{F}(1,8)=11, \mathrm{P}=0.011)$, a significant TMS x POSITION interaction $(\mathrm{F}(2,16)=6.1, \mathrm{P}=$ 0.011), and finally no significant HAND x TMS x POSITION interaction $(\mathrm{F}(2,16)=0.23, \mathrm{P}$ $=0.79)$.

A decomposition of the HAND x POSITION interaction revealed that RT decreased when the right or left hands were positioned in the right visual hemifield (normal and crossed positions respectively) and conversely, irrespective of the TMS location.

Figure 2 illustrates mean values $\pm 95 \% \mathrm{CI}$ for RT, in the right and left HAND conditions, and for the normal and crossed POSITION conditions.

Please insert figure 2 here

Using post-hoc comparisons, a first decomposition of the TMS $x$ POSITION interaction demonstrated a non-specific effect of TMS revealed by reduced simple reaction times (RT) with respect to the control condition both when applied to the parietal and frontal areas in the normal position conditions $(\mathrm{p}=0.010$ and $\mathrm{p}=0.007$ respectively for the right hand, and $\mathrm{p}=0.005$ and $\mathrm{p}=0.0004$ respectively for the left hand) but also when applied to the frontal area when hands were crossed ( $\mathrm{p}=0.0013$ for the right hand and $\mathrm{P}=0.0016$ for the left hand). Interestingly, mean RT (153 ms in the control condition) was decreased substantially by about $15 \mathrm{~ms}$. 
A consecutive decomposition of the TMS $x$ POSITION interaction revealed an increase of the RT when TMS was applied to the left parietal area when hands were crossed compared to hands in normal position, irrespective of the hand used $(p=0.004$ for the right hand and $\mathrm{p}=0.005$ for the left hand). We also computed effect sizes for this latter decomposition that illustrated the main result of our study and confirmed our statistical results. As expected, effect sizes were very low for the comparison between the Control TMS and normal POSITION vs. Control TMS and crossed POSITION ( $\mathrm{d}=0.008$ and $\mathrm{d}=0.02$ for the right and left HANDs respectively) as well as for the Frontal TMS and normal POSITION vs. Frontal TMS and crossed POSITION $(\mathrm{d}=0.010$ and $\mathrm{d}=0.014$ for the right and left HANDs respectively). By contrast, they were much higher for the comparison between the Parietal TMS and normal POSITION vs. Parietal TMS and crossed POSITION ( $\mathrm{d}=0.5$ and $\mathrm{d}=0.68$ for the right and left HANDs respectively). Importantly, these results were observed without any cueing and any constraints on movement selection since the response was imposed to the subjects, and without manipulation of the stimulus-response compatibility (single central visual stimulus).

Figure 3 illustrates mean values $\pm 95 \%$ CI for RT, in the normal and crossed POSITION conditions, and for the left frontal, left parietal and control TMS conditions.

Please insert figure 3 here

Finally, we also calculated the coefficient of variation. For this dependant variable, results of the ANOVA showed no significant main effect of $\operatorname{HAND}(\mathrm{F}(1,8)=4.7, \mathrm{P}=0.06)$, a significant main effect of TMS $(\mathrm{F}(2,16)=8.44, \mathrm{P}<0.003)$, a significant main effect of POSITION, i.e. CVs were superior when hands were crossed $(\mathrm{CV}=0.14 \pm 0.05$ and $0.16 \pm$ 0.06 respectively, $\mathrm{F}(1,8)=10.45, \mathrm{P}=0.012$ ), no significant HAND x TMS interaction 
$(\mathrm{F}(2,16)=1.59, \mathrm{P}=0.23)$, no significant HAND $\times$ POSITION interaction $(\mathrm{F}(1,8)=1.02, \mathrm{P}=$ 0.34), no significant TMS x POSITION interaction $(F(2,16)=0.07, P=0.93)$, and finally no significant HAND x TMS x POSITION interaction $(\mathrm{F}(2,16)=0.17, \mathrm{P}=0.85)$. Post-hoc comparisons revealed that the coefficient of variation was superior when the TMS was applied on the sham area in comparison to the frontal condition $(\mathrm{CV}=0.17 \pm 0.05$ and $0.13 \pm$ 0.05 respectively $\mathrm{P}<0.001)$ and in comparison to the parietal condition $(\mathrm{CV}=0.17 \pm 0.05$ and $0.14 \pm 0.05$ respectively $\mathrm{P}=0.002$ ). However, no significant difference was observed between the condition in which the TMS was applied on the frontal area in comparison to the parietal area condition $(\mathrm{P}=0.2)$. 


\section{Discussion}

When the arms of the subjects are crossed while their gaze is fixed straight ahead, visual information about hands position and arms proprioception are processed by different hemispheres. This situation may require some level of inter-hemispheric transfer and remapping. Our aim was to investigate whether a single pulse TMS applied to the left parietal cortex would increase simple visuo-manual RT for both hands in this situation. We only manipulated the hands position with respect to the saggital axis (normal vs. crossed) in order to focus on the inter-hemispheric remapping only and by minimizing attentional constraints. It is widely acknowledged that skills related to visuo-spatial attention involve large frontoparietal networks that present a right hemispheric specialization (Mesulam, 1981; Heilman and Van Den Abell, 1987; Halligan and Marshall, 1989). Consequently, in order to limit visuo-spatial bias induced by TMS and to focus on the inter-hemispheric remapping we decided to stimulate the left hemisphere and to design a task with a single central visual stimulus.

Our main results clearly demonstrated that RT increased when TMS was applied to the left parietal area when hands were crossed independently of the hand used. In addition, we observed that TMS can induce a non-specific effect and reduced simple RT with respect to the control condition. Irrespective of the stimulation condition, RT were shorter when the right or left hands were positioned in the right visual hemifield (normal and crossed positions respectively.

\subsection{The non-specific effect of TMS.}

We observed that TMS reduced simple reaction times (RT) with respect to the control condition either when applied to the parietal or frontal areas in the normal position conditions but also when applied to the frontal area when hands were crossed. Interestingly, mean RT 
(153 $\mathrm{ms}$ in the control condition) were decreased substantially by about $15 \mathrm{~ms}$ (on average). This non-specific effect of the TMS was already observed by Sawaki et al. (1999) in a similar simple reaction time paradigm in which TMS was applied at a subthreshlold level to the ipsilateral motor cortex or on the ipsilateral parietal cortex (left). More recently, Xu-Wilson et al. (2011) observed that a single pulse TMS applied to the head inhibited the ongoing saccade but was rapidly compensated for the perturbation. This effect was non-specific and independent of the TMS location. These authors suggested that TMS may produce synchronized stimuli of the startle system (Yeomans et al. 2002) and engage a circuit that affects the omni-pause neurones or the superior colliculus. In accordance, several studies have demonstrated that the superior colliculus is sensible to electro-sensory stimuli (Stein et al. 1975, Hartline et al. 1978, Knudsen et al. 1982, Meredith and Stein 1983, King and Palmer 1985). Moreover, Bischoff et al. (1993) suggested that magnetic stimulation of the supraorbital nerve may be an alternative way to study the blink and the startle reflexes. Interestingly, results also demonstrated that the coefficients of variation decreased similarly for the frontal (F3) and parietal (P3) TMS conditions. This suggests that response variability decreased when TMS was applied. This result mirrors the non-specific effect of TMS and may reflect the startle-like reflex response observed with TMS that may synchronize motor responses.

Altogether, it suggests that a startle-like reflex may be superimposed and precede the normal motor responses with the TMS. This rapid response is independent from the auditory signal associated to the magnetic pulse.

\subsection{The effect of hand position in the visual field.}

RT increased when a single pulse TMS was applied to the left parietal area and when hands were crossed independently of the hand used. It suggests that inter-hemispheric remapping 
between somato-sensory information and vision of the hand, is mediated by the parietal cortex. Interestingly, for goal directed movements, Rossetti et al. (1995) have demonstrated that the vision of the hand prior to the movement was processed to improve the rate of terminal error (Rossetti et al. 1995). When no hand displacement is required we demonstrated that the vision of the hand in the opposite hemifield is still processed. Before the go-signal, subjects were instructed the hand to use for the motor response. Despite the motor program is very simple and already selected and anticipated, peripheral visual cues about the hand position may be still processed. This is all the more surprising that the movement is not goal directed and processing constraints are minimal in our task (as sustained by the very short reactions times we measured especially in the conditions with TMS). In consequence, the brain does not deal with a single sensory modality and inter-hemispheric remapping is required when arms are crossed in this simple task. In accordance, it has been shown that optic ataxia, a specific visuo-manual guidance deficit associated to a lesion of the parietal cortex, is not only optic but also involves impairments in the spatial integration of proprioceptive information (Blangero et al. 2007).

Importantly, we also did not cue the motor responses and did not impose any constraints on movement selection since the hand choice was imposed to the subjects before the response and blocked for several trials. In addition, the stimulus-response compatibility was not manipulated. In consequence, this remapping was not distracted by any sensory cues nor influenced by any processes (e.g. memory, decision making or visual attention to the stimulus).

The interaction between the hand and the position revealed that RT decreased when the right or left hands were positioned in the right visual hemifield (normal and crossed positions respectively) and conversely, irrespective of the TMS condition. However, it is well known that right hemisphere dominance for visuospatial attention, produces a slight leftward 
deviation of attention in healthy individuals (Mesulam 1981) responsible for a leftward bias in line bisection (Milner et al. 1992). This bias is called 'pseudoneglect' (Jewell and McCourt 2000) in comparison with the large rightward bias observed in neglect (Mesulam 1981). This hemispheric dominance appears early during childhood (Dellatolas et al. 1996). We obtained somewhat different results that suggest a clear dominance of the visual attention directed towards the effector or the limb positioned in the right hemifield. This may be particularly true when the visual stimulus is not manipulated and remain the same. In consequence, we suspect that some motor attention (James 1890, Rushworth et al. 1997) is mixed with visual attention and that there may be a component of visual attention that is also directed towards the effector (and not the stimulus) with predominance for the right hemifield. Interestingly, Rushworth et al. (1997) have emphasized the participation of the left parietal cortex in motor attention.

In conclusion, sensori-motor remapping may be influenced by the hand position in the visual field when hands are crossed together. This effect may depend on somatosensory and visual inputs. However, it is also combined with a clear dominance in favor of some visual attention directed towards the hand located on the right. In the context of clinical practice, (r)TMS can be used to modulate brain activity during rehabilitative motor tasks by selectively interfering with the activity of one hemisphere with respect to the other (Hummel and Cohen 2006) However, when the hands are crossing the saggital axis it should be kept in mind that some inter-hemispheric remapping can be involved. Consequently, (r)TMS could have bilateral effects whereas the first clinical intention would be to have inhibitory or excitatory effects on one hemisphere or hemibody. Nevertheless, it does not mean that arm crossing paradigms are useless in rehabilitation.

Acknowledgments: we thank the three anonymous reviewers for their stimulating comments about the present work and Anirban Dutta for careful reading of the manuscript. 


\section{References}

Andersen RA (1995) Encoding of intention and spatial location in the posterior parietal cortex. Cereb Cortex 5(5):457-69.

Azanon E, Camacho K, Soto-Faraco S. (2010) Tactile remapping beyond space. Eur J Neurosci 31: 1858-1867.

Bermpohl F, Fregni F, Boggio PS, Thut G, Northoff G, Otachi PT, Rigonatti SP, Marcolin MA, Pascual-Leone A (2005) Left prefrontal repetitive transcranial magnetic stimulation impairs performance in affective go/no-go task. Neuroreport. 2005 Apr 25;16(6):615-9.

Bischoff C, Liscic R, Meyer BU, Machetanz J, Conrad B (1993) Magnetically elicited blink reflex: an alternative to conventional electrical stimulation. Electromyography and Clinical Neurophysiology 33(5):265-9.

Blangero A, Ota H, Delporte L, Revol P, Vindras P, Rode G, Boisson D, Vighetto A, Rossetti Y, Pisella L (2007) Optic ataxia is not only 'optic': impaired spatial integration of proprioceptive information. Neuroimage 36(2):T61-8.

Bolognini N, Maravita A (2007) Proprioceptive alignment of visual and somatosensory maps in the posterior parietal cortex. Current Biology 6;17(21):1890-5.

Boorman ED, O'Shea J, Sebastian C, Rushworth MF, Johansen-Berg H (2007) Individual differences in white-matter microstructure reflect variation in functional connectivity during choice. Curr Biol. 21;17(16):1426-31. 
Buchholz VN, Goonetilleke SC, Medendorp WP, Corneil BD (2012) Greater benefits of multisensory integration during complex sensorimotor transformations. J Neurophysiol. (11):3135-43.

Buneo CA, Jarvis MR, Batista AP, Andersen RA (2002) Direct visuomotor transformations for reaching. Nature 11;416(6881):632-6.

Cohen YE, Andersen RA (2002) A common reference frame for movement plans in the posterior parietal cortex. Nature Review Neuroscience 3(7):553-62.

Dellatolas G, Coutin T, De Agostini M (1996) Bisection and perception of horizontal lines in normal children. Cortex 32:705-715.

Desmurget, M; Epstein, CM; Turner, RS; et al. (1999) Role of the posterior parietal cortex in updating reaching movements to a visual target. Nat Neurosci 6:563-567.

Duhamel JR, Bremmer F, BenHamed S, Graf W (1997) Spatial invariance of visual receptive fields in parietal cortex neurons. Nature 389(6653):845-8.

Duhamel JR, Colby CL, Goldberg ME (1992) The updating of the representation of visual space in parietal cortex by intended eye movements. Science 3;255(5040):90-2.

Hartline PH, Kass L, Loop MS (1978) Merging of modalities in the optic tectum: Infrared and visual integration in rattlesnakes. Science 199(4334):1225-9. 
Halligan PW, Marshall JC (1989). Line bisection in visuo-spatial neglect: Disproof of a conjecture. Cortex. 25, 517-521.

Heilman KM, Van Den Abell T (1979). Right hemispheric dominance for mediating cerebral activation. Neuropsychologia. 17:315-21.

Herwig U, Schönfeldt-Lecuona C, Wunderlich AP, von Tiesenhausen C, Thielscher A, Walter H, Spitzer M. (2001) The navigation of transcranial magnetic stimulation.

Psychiatry Res. 108(2):123-31.

Herwig U, Satrapi P, Schönfeldt-Lecuona C (2003) Using the international 10-20 EEG system for positioning of transcranial magnetic stimulation. Brain Topogr. 16(2):95-9.

van den Heuvel OA, Van Gorsel HC, Veltman DJ, Van Der Werf YD (2013) Impairment of executive performance after transcranial magnetic modulation of the left dorsal frontal-striatal circuit. Hum Brain Mapp.;34(2):347-55.

Goldman-Rakic PS (1987) Motor control function of the prefrontal cortex. Ciba Found Symp. 132:187-200. Review.

Griskova I, Ruksenas O, Dapsys K, Herpertz S, Hoppner J. (2007) The effects of $10 \mathrm{~Hz}$ transcranial magnetic stimulation on resting EEG power spectrum in healthy subjects. Neuroscience Letters 2007;419(2):162-167.

Head H, Holmes G (1911) Sensory disturbance from cerebral lesions. Brain 34(2-3): 102-254 
Heed T, Roder B (2010) Common anatomical and external coding for hands and feet in tactile attention: evidence from event-related potentials. J Cogn Neurosci 22: 184-202.

James W (1890) Principles of Psychology, 1st edition. Holt, New York.

Jewell G, McCourt ME (2000) Pseudoneglect: a review and meta-analysis of performance factors in line bisection tasks. Neuropsychologia 38(1):93-110.

King AJ, Palmer AR (1985) Integration of visual and auditory information in bimodal neurones in the guinea-pig superior colliculus. Experimental Brain Research 60(3):492-500.

Knudsen EI (1982). Auditory and visual maps of space in the optic tectum of the owl. Journal of Neuroscience 2(9):1177-94.

Lloyd DM, Shore DI, Spence C, Calvert GA (2003) Multisensory representation of limb position in human premotor cortex. Nature Neuroscience 6(1):17-8.

Marzi CA, Bisiacchi P, Nicoletti R (1991) Is interhemispheric transfer of visuomotor information asymmetric? Evidence from a meta-analysis. Neuropsychologia 29:1163-1177

Marzi CA, Miniussi C, Maravita A, Bertolasi L, Zanette G, Rothwell JC, Sanes JN (1998) Transcranial magnetic stimulation selectively impairs interhemispheric transfer of visuomotor information in humans. Exp Brain Res.118(3):435-8. 
Meredith MA, Stein BE (1983) Interactions among converging sensory inputs in the superior colliculus. Science 221(4608):389-91.

Mesulam MM (1981) A cortical network for directed attention and unilateral neglect. Annals of Neurology 10(4):309-25.

Milner AD, Brechmann M, Pagliarini L (1992) To halve and to halve not: an analysis of line bisection judgements in normal subjects. Neuropsychologia 30(6):515-26.

Oldfield RC (1971) The assessment and analysis of handedness: the Edinburgh inventory. Neuropsychologia 9:97-113.

Overvliet KE, Azanon E, Soto-Faraco S (2011) Somatosensory saccades reveal the timing of tactile spatial remapping. Neuropsychology 49: 3046-3052.

Petrides M, Milner B (1982) Deficits on subject-ordered tasks after frontal- and temporal-lobe lesions in man. Neuropsychologia. 20(3):249-62.

Rossetti Y, Desmurget M, Prablanc C (1995) Vectorial coding of movement: vision, proprioception, or both? J Neurophysiol. 74(1):457-63.

Rushworth MF, Nixon PD, Renowden S, Wade DT, Passingham RE (1997) The left parietal cortex and motor attention. Neuropsychologia 35(9):1261-73. 
Sawaki L, Okita T, Fujiwara M, Mizuno K (1999) Specific and non-specific effects of transcranial magnetic stimulation on simple and go/no-go reaction time. Experimental Brain Research 127(4):402-8.

Schutter D, van Honk J. (2006) Increased positive emotional memory after repetitive transcranial magnetic stimulation over the orbitofrontal cortex. J. Psychiatry Neurosci 31(2):101-104.

Stein BE, Magalhães-Castro B, and Kruger L (1975) Superior colliculus: Visuotopicsomatotopic overlap. Science 189 (4198):224-6.

Thiebaut de Schotten M, Urbanski M, Duffau H, Volle E, Lévy R, Dubois B, Bartolomeo P (2005) Direct evidence for a parietal-frontal pathway subserving spatial awareness in humans. Science 30;309(5744):2226-8. Erratum in: Science (2007) 317(5838):597.

Wassermann E, Epstein C and Ziemann U (2002) Oxford Handbook of Transcranial Stimulation. Oxford: Oxford University Press. pp. 13-23, 25-32. ISBN 0-19-856892-4.

Xu-Wilson M, Tian J, Shadmehr R, Zee DS (2011) TMS perturbs saccade trajectories and unmasks an internal feedback controller for saccades. Journal of Neuroscience 31(32):1153746.

Yeomans JS, Li L, Scott BW, Frankland PW (2002) Tactile, acoustic and vestibular systems sum to elicit the startle reflex. Neuroscience and Biobehavioral Review 26(1):1-11. 


\section{Figures captions}

Figure 1. Experimental set-up of the simple visuo-manual reaction time task in the normal (i.e. uncrossed) hands position.

Figure 2. RT for the left and right hand in normal and crossed conditions:

Mean values $\pm 95 \%$ CI for RT, in the right and left HAND conditions, and for the normal and crossed POSITION conditions.

Figure 3. Effects of the three TMS conditions on the RT in normal and crossed positions:

Mean values $\pm 95 \%$ CI for RT, in the normal and crossed POSITION conditions, and for the left frontal, left parietal and control TMS conditions. 